\title{
Alternative lengthening of telomeres is induced by telomerase inhibitors in Barrett's esophageal cells
}

\author{
XIUGUANG QIN, BO QI and BAOSHENG ZHAO \\ Department of Thoracic Surgery, The First Affiliated Hospital of Xinxiang \\ Medical University, Weihui, Henan 453100, P.R. China
}

Received August 29, 2012; Accepted December 10, 2012

DOI: $10.3892 / o r .2013 .2238$

\begin{abstract}
A crucial step in the path to the malignant transformation of cells and tumor formation is immortalization, which essentially depends on telomere maintenance. The aim of this study was to investigate the role of telomerase in the progression of Barrett's esophagus. Telomerase activity was measured in Barrett's cells using terminal restriction fragment (TRF) analysis. Telomere length was measured using Q-FISH analysis. Furthermore, the telomere recombination events were detected between sister chromatids using chromosome orientation FISH (CO-FISH). There was a reduction in telomerase activity in the CP-A cells transduced with MT-hTER/47A+siRNA, which led to an almost complete disappearance of telomerase activity. The telomere length of the CP-A cells transduced with MT-hTER/47A+siRNA was slightly shorter compared to that of the untransduced cells. The telomerase-inhibited cells were morphologically indistinguishable from those untransduced and WT-hTER-transduced cells. In the control cells, the growth rate was between 0.9 to 1.1 with the population doubling per day. Although the transduction of the telomerase inhibitors in the CP-A cells did not cause a significant reduction in cell growth, these transduced cells grew generally slower compared with the control cells. The heterogeneous telomere length was also be detected in the telomerase-inhibited CP-A cells. However, the telomere length remained homogeneous in the control cells. The metaphase of the CP-A cells transduced with MT-hTER/47A+siRNA demonstrated $70 \%$ heterogeneous telomeres. In addition, no increased recombination was observed between sister chromatids in the transduced CP-A cells compared with the control cells. Our findings suggest that an alternative lengthening of telomeres (ALT) may be induced by telomerase inhibitors in CP-A cells. Therefore, telomerase inhibitors may exhibit
\end{abstract}

Correspondence to: Dr Baosheng Zhao, Department of Thoracic Surgery, The First Affiliated Hospital of Xinxiang Medical University, Weihui, Henan 453100, P.R. China

E-mail: scilwok@foxmail.com; kitiyanant@163.com

Key words: telomerase, Barrett's esophagus, telomerase activity, alternative lengthening of telomeres, telomerase inhibitor high potency in the treatment of esophageal adenocarcinoma arising from Barrett's esophagus.

\section{Introduction}

Normal human cells have a limited life span due to the loss of telomeric sequences after each DNA replication (1). A critically short telomere length causes the cells to move into the stage of senescence and ultimately to pursue the path of apoptosis (2). This mechanism helps to protect the entire organism (3). Early studies of hTERT expression revealed that telomerase is suppressed in human somatic tissues, but it is expressed robustly in germ and tumor cells (4). However, germ and embryonic cells are able to maintain their telomere stability to clone adult cell nuclei into their shortened telomeres $(5,6)$. Telomerase activity alone is not enough to maintain stable telomere lengths, since telomeres continue to shorten $(7,8)$. Even hTERT expression and telomerase activation in early passages of human fibroblasts are mainly detected in the $\mathrm{S}$ phase (9). This indicates that the regulation of hTERT and telomerase in normal cells is important for the proliferation of normal human cells.

Evidence for the role of telomeres in tumorigenesis has been provided in studies of primary human fibroblasts. These primary human cells reach a limited potential of replication of approximately $60-80 \%$ population doubling per day before moving into the senescence stage (10). In contrast, tumor cells establish indefinite devision in culture. Observations of primary and tumor cells indicate that this difference was associated with the shortening of telomeres. There is a loss of telomeric sequences in normal primary cells after each cell division, but not in tumor cells (1). However, tumor cells achieve a stabilization of telomeres through the activation of telomerase or alternative lengthening of telomere (ALT) mechanism, and thus overcome the senescence stage. Immortalization is an important prerequisite for unimpeded tumor growth and thus is an essential step in the malignant transformation of cells (11). Thus, immortalization allows an unhindered proliferation of tumor cells and malignant transformation by the accumulation of genetic alterations.

The ALT mechanism plays an important role in malignant transformation. In a carcinogenesis model, cell cycle immortalization based on ALT induced an oral-esophageal squamous epithelial origin after inactivation of the tumor-suppressor 
Table I. List of the plasmids used for trasduction.

\begin{tabular}{lll}
\hline ID & \multicolumn{1}{c}{ Name } & \\
\hline Plasmid 1 & WT-hTER & Wild-type RNA template \\
Plasmid 2 & MT-hTER/AU5 & Mutated RNA template \\
Plasmid 3 & MT-hTER/47/A & Mutated RNA template \\
Plasmid 6 & MT-hTER/AU5+siRNA & Mutated RNA template in combination with an siRNA against the RNA template \\
Plasmid 7 & MT-hTER/47A+siRNA & Mutated RNA template in combination with an siRNA against the RNA template \\
Plasmid 8 & Empty vector & Control
\end{tabular}

p53 and overexpression of cyclin D1 protein (12). In addition, overexpression of epidermal growth factor receptor (EGFR) resulted in the in vitro transformation of these cells through concomitant activation of telomerase $(13,14)$. Since a crucial step in the pathway to malignant transformation of cells and tumor formation is immortalization, which essentially depends on telomere maintenance, the present study aimed to investigate the role of telomerase in the progression of esophageal adenocarcinoma arising from Barrett's esophagus.

\section{Materials and methods}

Cell line and culture. The Barrett's esophageal cell line CP-A was used in this study. CP-A cells were cultured in serumfree keratinocyte medium (Invitrogen Life Technologies) and passaged when reaching $30-40 \%$ confluence. Subsequently, the medium was removed, and the cells were washed with $5 \mathrm{ml}$ PBS and resolved with a Trypsin/PBS mixture (1:1). After a 10- to 20-min incubation in an incubator at $37^{\circ} \mathrm{C}$ in $5 \% \mathrm{CO}_{2}$, the dissolved cells were taken up in $6 \mathrm{ml}$ medium containing Dulbecco's modified Eagle's medium F-12 (DMEM; Invitrogen) and centrifuged for $5 \mathrm{~min}$ at $138 \mathrm{x} \mathrm{g}$. Subsequently, the supernatant was discarded, and the cells were diluted to 1:10-1:80 in fresh medium according to the thickness of the cell pellets. These cells were then transferred into new $10-\mathrm{cm}$ culture dishes. The cells were maintained in an incubator at $37^{\circ} \mathrm{C}$ in $5 \% \mathrm{CO}_{2}$ in a moisture-saturated atmosphere. In order to wash the cells, only the medium was removed. The cells were washed with $5 \mathrm{ml}$ of PBS, and 8-10 $\mathrm{ml}$ of fresh cells suitable for the respective medium was added.

DNA extraction. DNA extraction was performed using the QIAamp ${ }^{\circledast}$ DNA Mini kit (Qiagen) according to the manufacturer's protocol. After trypsinization and subsequent centrifugation, the cells were resuspended in $1 \mathrm{ml}$ of PBS and centrifuged for $1 \mathrm{~min}$ at $5.9 \mathrm{x}$ g. The entire supernatant was carefully removed, and the pellet was resuspended in $200 \mu \mathrm{l}$ PBS. This was followed by addition of $20 \mathrm{ml}$ proteinase $\mathrm{K}$ (Qiagen) and lysis buffer AL (Qiagen), and mixed for $15 \mathrm{sec}$ and then incubated at $56^{\circ} \mathrm{C}$ for $10 \mathrm{~min}$. After incubation, the tubes were centrifuged briefly, and $200 \mu 1$ 100\% ethanol was added. The mixtures were mixed again for $15 \mathrm{sec}$ and finally applied to a well QIAamp Mini spin column and centrifuged at $5.8 \mathrm{x}$ g for $1 \mathrm{~min}$. The column was placed in a new collection tube, $500 \mu \mathrm{l}$ of AW1 buffer (Qiagen) was added to the column and centrifugation was carried out for $1 \mathrm{~min}$ at $5.9 \mathrm{x}$ g. Then $500 \mu \mathrm{l}$ AW2 buffer (Qiagen) was added to the column and centrifugation was carried out for $3 \mathrm{~min}$ at $15.7 \mathrm{x} \mathrm{g}$. DNA was placed in a $1.5-\mathrm{ml}$ reaction vessel, pipetted with 150-200 $\mu \mathrm{l}$ AE buffer (Qiagen) and centrifuged for 1 min with incubation at RT at $5.8 \mathrm{xg}$.

Transduction. The cells were seeded in a 6-well plate at $2.5 \times 10^{5} /$ well and incubated overnight in an incubator at $37^{\circ} \mathrm{C}$ in $5 \% \mathrm{CO}_{2}$. Two days later the cells were washed and spread onto a 6-well plate with the infection medium containing $2 \mathrm{ml}$ of optimal media and $8 \mathrm{mg} / \mathrm{ml}$ Polybrene ${ }^{\circledR}$. The cells were then centrifuged for $2 \mathrm{~h}$ at $800 \times \mathrm{g}$ at $20^{\circ} \mathrm{C}$, and then incubated overnight in an incubator at $37^{\circ} \mathrm{C}$ in $5 \% \mathrm{CO}_{2}$. On the next day, the infection medium was removed and replaced with $2 \mathrm{ml}$ of fresh medium. A few days after transduction, when cells were grown to a sufficient density, the cells were analyzed and sorted using a MoFlo high-speed cell sorter (Dako). The cells were then trypsinized, centrifuged and resuspended in $1 \mathrm{ml}$ of DMEM and PBS and maintained on ice during the sorting process. After sorting, the cells were added to a fresh $10 \mathrm{~cm}$ plate, which was filled with fresh medium. The plasmids used for transduction are listed in Table I.

Terminal restriction fragment (TRF) analysis. The DNA digestion was performed using the RsaI/HinfI (Roche, Indianapolis, IN, USA) restriction enzymes. The restriction mixture $(50 \mathrm{ml})$ contained 2-5 g DNA, 1X NEB buffer 2 or 4, $10 \mathrm{U}$ of $R s a \mathrm{I}$ and $10 \mathrm{U}$ of HinfI.

The DNA extraction was digested overnight at $37^{\circ} \mathrm{C}$. Each $4 \mu \mathrm{l}$ of digested or undigested DNA was mixed with $16 \mu \mathrm{l} \mathrm{TE}$ buffer (Qiagen) and 41 6X loading buffer (Fermentas), loaded alternately onto a $1 \%$ agarose TAE gel, boiled in a microwave oven and treated with $0.1 \mu \mathrm{g} / \mathrm{ml}$ ethidium bromide. The mixture was poured into cooled horizontal gel chambers for $1 \mathrm{~h}$. The separation time was $1 \mathrm{~h}$ at $120 \mathrm{~V}$. Then it was poured in a Protean XL horizontal chamber (Bio-Rad Laboratories). Samples (20 ml) were mixed with $4 \mu 16 \mathrm{X}$ loading buffer. The running time was $14 \mathrm{~h}$ at $40 \mathrm{~V}$. The gel was then viewed under UV light. In preparation for the Southern blot analysis, the gel was placed for $15 \mathrm{~min}$ in $0.25 \mathrm{M}$ hydrochloric acid and finally washed with neutralizing buffer. Southern blot analysis was performed.

Fluorescence in situ hybridization. The quantitative fluorescence in situ hybridization (Q-FISH) was performed by metaphase chromosome spread preparation with a telomeric PNA probe, which was labeled with the fluorescent dye $\mathrm{Cy} 3$ hybridized. After counterstaining the chromosomes with DAPI, they were observed under a fluorescence micro- 


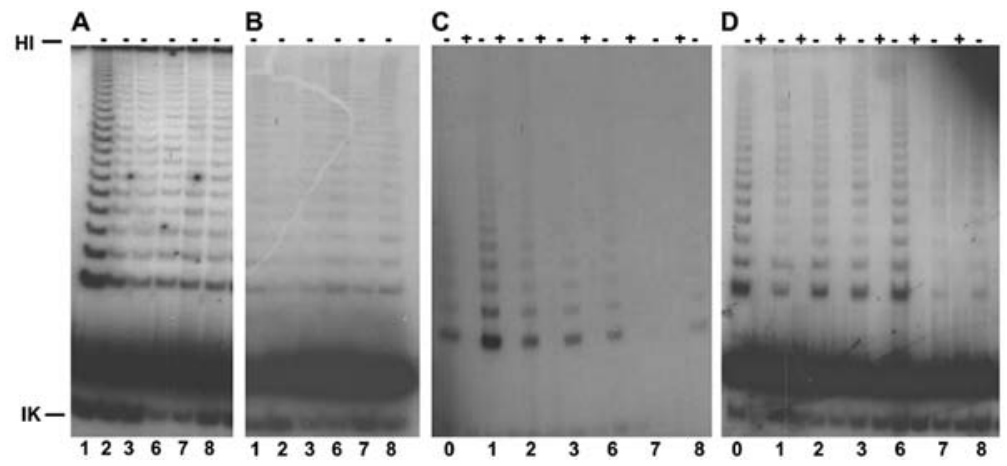

Figure 1. Telomerase activity in passage 32 (A), 50 (B), 86 (C) and 109 (D) of telomerase-inhibited CP-A and control cells.
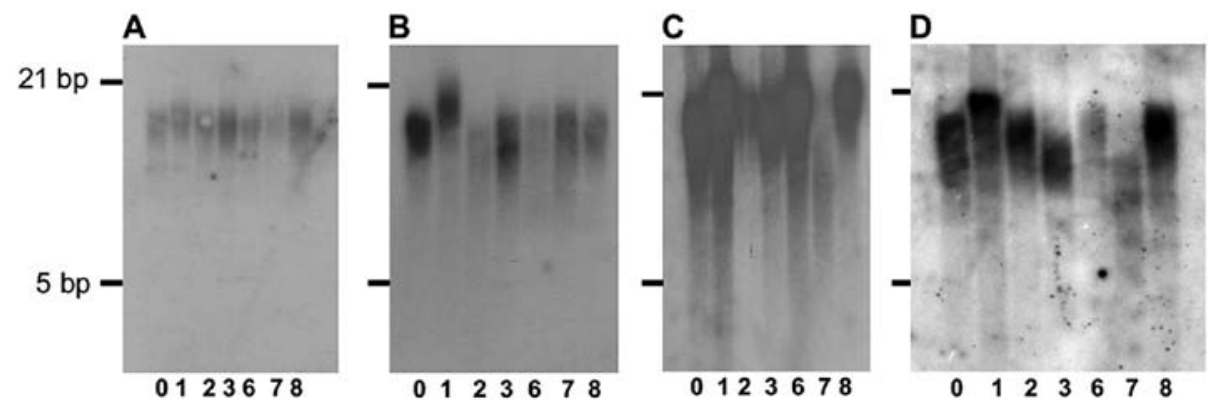

Figure 2. Telomere length determination in passage 32 (A), 50 (B), 86 (C) and 109 (D) of telomerase-inhibited CP-A and control cells using terminal restriction fragment (TRF) analysis.
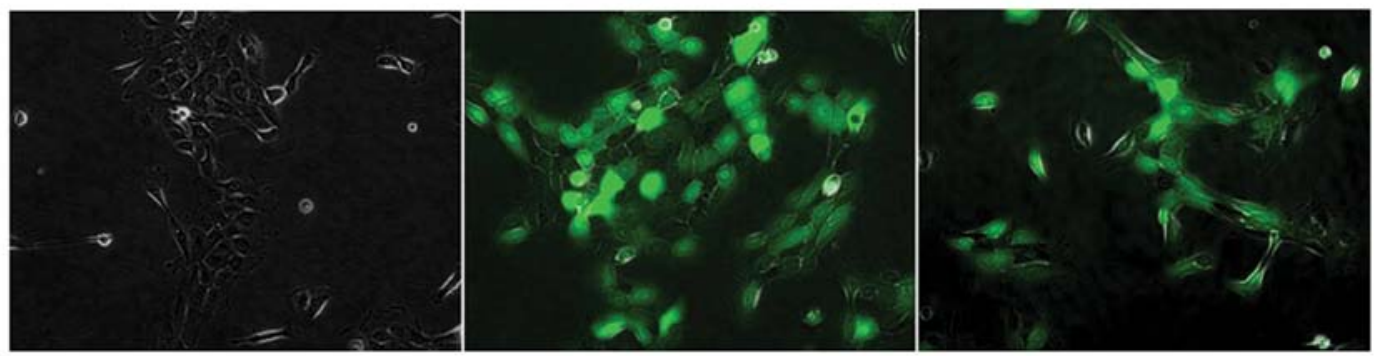

Figure 3. Fluorescence microscopy images of the untransduced (left), WT-hTER- (middle) and MT-hTER/47A+siRNA-transduced (right) CP-A cells

scope and the fluorescence intensities of the telomeres was determined. The advantage of this method is that telomere measurements may be performed on individual cells. Q-FISH method was performed as previously described (15). In chromosome orientation FISH (CO-FISH), the exchange of sister chromatids at telomeres and chromosomes may be demonstrated in single cells.

\section{Results}

Telomerase inhibition was observed in the genetically transduced Barrett's and control cells with telomerase inhibitors. These cells were transduced with the telomerase inhibitors at a very low initial passage (passage 32 ).

There was a reduction in telomerase activity after CP-A cells were transduced with plasmid 7 , which led to an almost complete disappearance of telomerase activity. This inhibition was demonstrated in different passages (Fig. 1).
The telomere length of the control cells was reduced compared with the untransduced wild-type CP-A cells as determined by TRF analysis. The telomeres were stable and were $12 \mathrm{kbp}$ in 3 passages after transduction with plasmids 1-8 (Fig. 2A). After transduction with plasmid 1 (WT-hTER), analysis of the telomere lengths at passage 50 demonstrated an extension of telomeres, which was ultimately $>21 \mathrm{kbp}$.

The telomere length of the telomerase-inhibited cells initially remained the same length as the control cells, although a significant reduction in telomerase activity was noted after transduction with plasmid 7 (MT-hTER/47A) over further passages $36 \mathrm{~h}$ later. The telomere length of the telomerase-inhibited cells remained the same length as the control cells in passage 86 (Fig. 2C). In contrast, the telomere lengths of the CP-A cells transduced with plasmid 7 were slightly shorter (Fig. 2C). This telomere heterogeneity remained constant over a further passage 23 (Fig. 2D), indicating an ALT mechanism. 


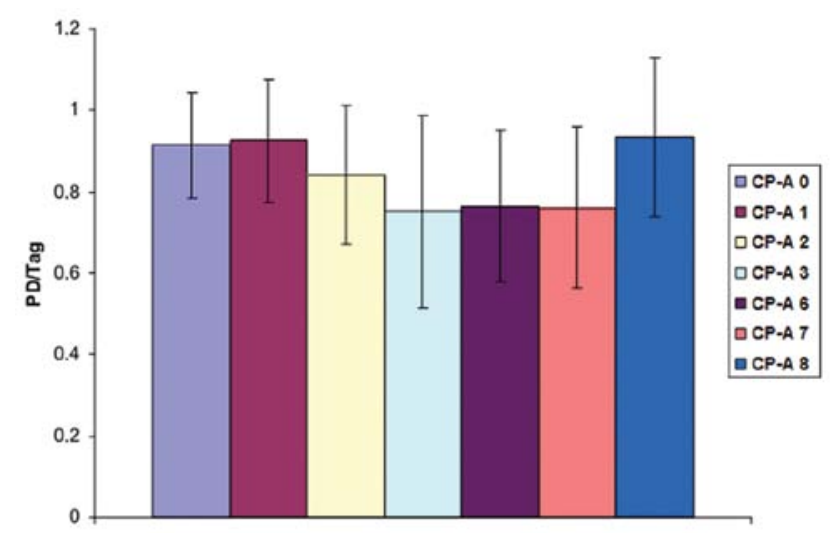

Figure 4. Growth behavior of the CP-A cells.

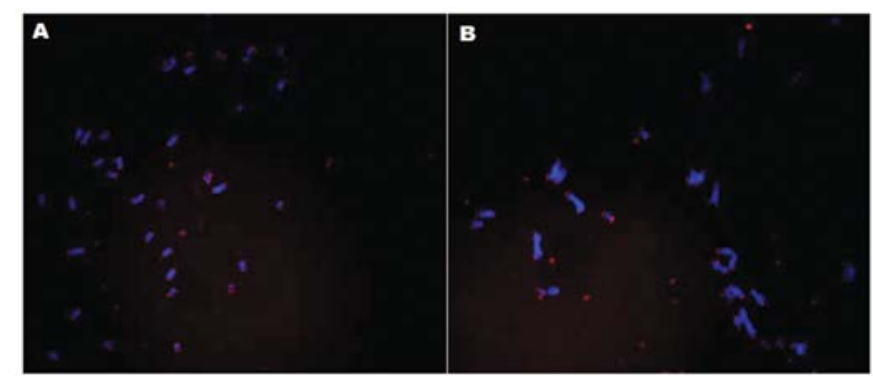

Figure 5. Sample images of homogeneous telomere length determination in control cells (A) and heterogeneous telomere length in the MT-hTER/47A+siRNA transduced CP-A cells (B) using Q-FISH analysis.

Following the successful reduction in telomerase by the telomerase inhibitory plasmids, the cells were examined for morphological changes. The different telomerase-inhibited cells were morphologically indistinguishable from the untransduced and WT-hTER-transduced cells (Fig. 3). The cells underwent division normally and showed no cellular morphology for further changes of apoptosis. The GFP-positive cells differed only in their brightness.

To create the growth curve of the transduced CP-A cells, CP-A cells transduced with plasmid 1-8 were plated at $1 \times 10^{5}$ cells/well and trypsinized for 2-3 days. Then the cells were counted. This procedure was repeated 4 to 6 times. In the untransduced cells and the cells transduced with plasmid 1 or 8 , the growth rate was between 0.9 to 1.1 with the population doubling per day (Fig. 4). A similar situation was also noted in the CP-A cells transduced with telomeraseinhibitory plasmids 2-7. Again, the transduction with the telomerase inhibitors did not cause a significant reduction in cell growth. However, these transduced cells grew generally slower compared with the untransduced cells and the cells transduced with plasmid 1 or 8 .

A heterogeneous telomere length was also detected in the telomerase-inhibited CP-A cells (Fig. 5B). However, the telomere length remained homogeneous in the control cells (Fig. 5A). The untransduced cells and the cells transduced with plasmid 1-8 were seeded for $6 \mathrm{~h}$, and on the next day cells were treated with colcemid to arrest cells in the metaphase. After the $\mathrm{KCl}$ treatment and subsequent fixation, the swollen

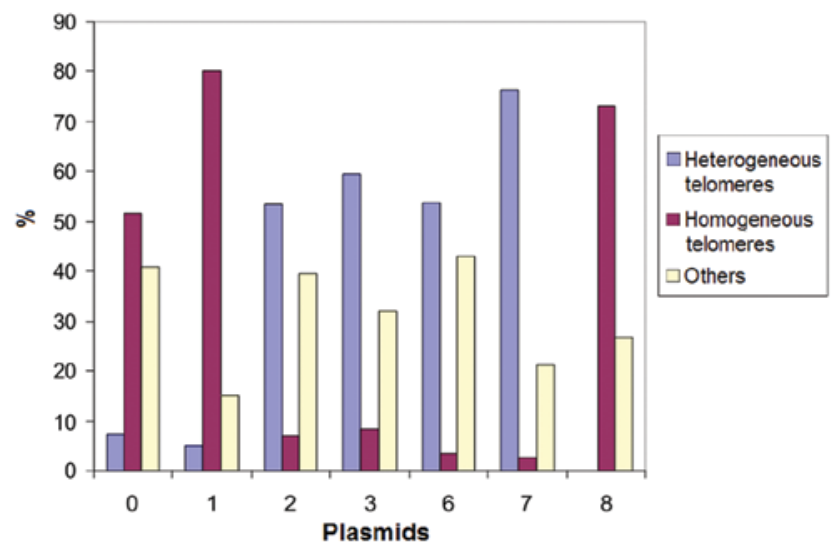

Figure 6. Q-FISH analysis of telomerase inhibitor-transduced CP-A cells

cells were dropped onto a slide and thus burst. The metaphase spreads were stained with DAPI (blue) and the telomeres with a Cy3-labeled probe (red), and the telomeric sequence was hybridized.

The metaphases of CP-A cells transduced with plasmid 7 showed $70 \%$ heterogeneous telomeres (Fig. 6). This correlated with the decrease in telomerase activity in CP-A cells transduced with plasmid 7 as determined by TRF analysis and the increasing heterogeneous telomere lengths.

To investigate the exchange of sister chromatids, the chromosome orientation FISH (CO-FISH) was performed. The newly replicated DNA strand was degraded by metaphase, leading to a single-stranded target DNA with either a C-rich or G-rich telomere strand. The next step was the hybridization with specific probes, performed either against the C-rich (green) and G-rich (red) strand. In the event there was a recombination between sister chromatids, a colocalization of two probes was found at the telomeres, which led to a yellow color.

However, no increased recombination was observed between sister chromatids in the transduced CP-A cells (Fig. 7B) compared with the control cells (Fig. 7A). Thus, there existed an ALT mechanism that was not based directly on the exchange of sister chromatids.

\section{Discussion}

All telomerase-inhibited cells were compared with the untransduced and plasmid 1- and 8-transduced cells. To confirm whether the telomerase inhibition led to a reduction in telomerase, the enzyme activity was determined. A reduction in telomerase was observed in all CP-A cells transduced with the telomerase-inhibiting plasmids, similar to that noted in other tumor cells after telomerase inhibition (16). This inhibition remained stable. There was an increased telomerase inhibition in Barrett's cells after transduction with plasmid 7, which is a combination of mutated RNA template and an siRNA against the RNA template. This is considered logical, since the mutated version is present in the cells and the RNA template is present and may be inserted in the telomerase, thus leading to altered telomere sequences. In contrast, endogenous RNA can be inhibited by the complementary siRNA. The two 
A

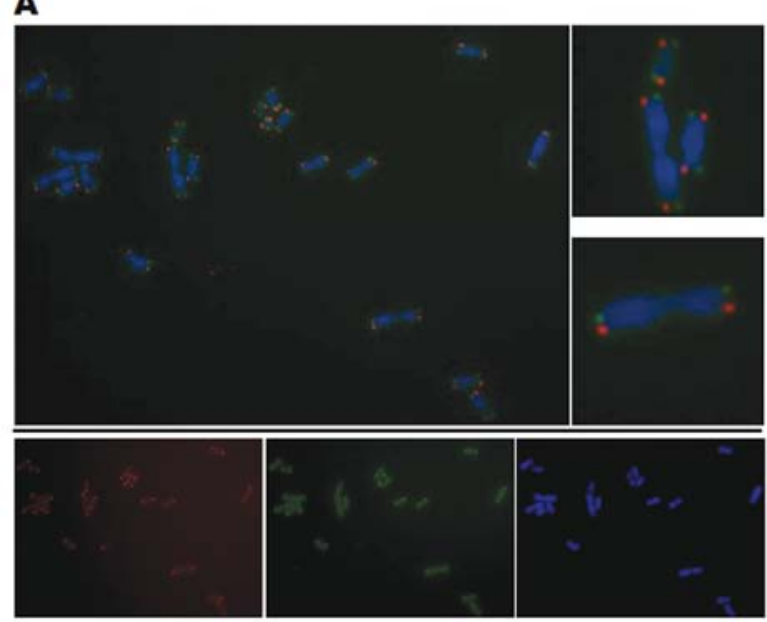

B

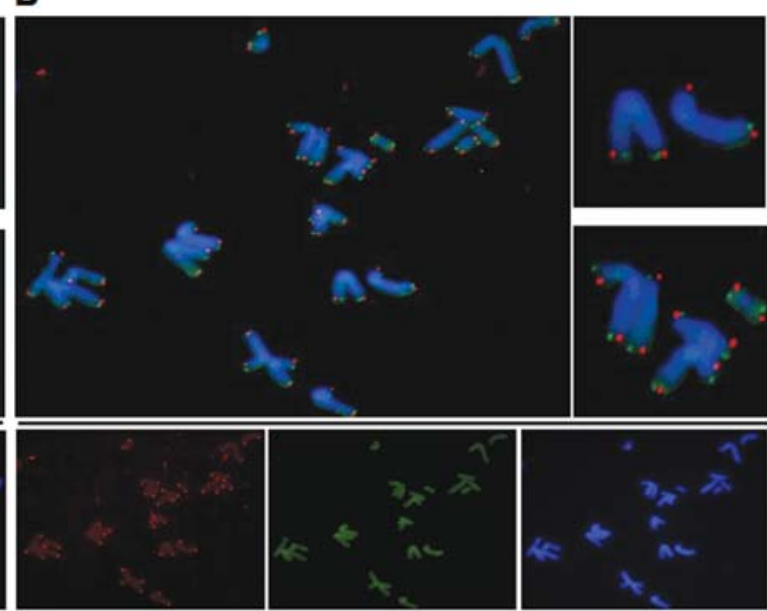

Figure 7. CO-FISH analysis of CP-A cells. (A) Untransduced CP-A cells; (B) MT-hTER/47A+siRNA-transduced CP-A cells. The C-rich strand is red, the G-rich stramd is green and the chromosomes are blue.

plasmids (6 and 7) were also found to cause almost complete inhibition of growth in the HCT116 colon carcinoma cell line with telomerase inhibition after 10 days (16).

After transduction with the control plasmid 1, increased telomerase activity was observed. This amplification of telomerase activity by exogenous overexpression of hTER was also observed in other tumor cells, such as HeLa cells and HT1080 cells (17). Previously it was shown in various species that the cells were inhibited in their growth after telomerase inhibition $(16,18-20)$, while the telomere lengths did not decrease (16). The telomere lengths were observed over many passages in the Barrett's cells. The first stable telomere length was shorter in Barrett's cells after transduction with the telomerase inhibitors. Heterogeneous telomere lengths are an essential characteristic that results following the activation of the ALT mechanism (21). It has also been shown that immortalized esophageal keratinocytes maintain their telomere lengths in spite of the reduced telomerase activity after telomerase inhibition (22).

In the Barrett's cells, the telomere lengths were increased after transduction with plasmid 1. Researchers have observed that the exogenous expression of hTERT and telomerase activity led to increased and lengthened telomeres in hTERoverexpressing HT1080 cells and HeLa cells (23). However, the telomere lengths were noted with the increasing population doubling per day of a heterogeneous character, as described in ALT cells (17). The telomeres were lengthened, but only up to a certain length, which was not further exceeded. This may be because telomerase-positive cells receive a balance between telomere erosion and erect extension. This is a negative feedback by telomere length on telomerase activity, where each individual telomere is regulated by telomere-binding protein. Thus, a plateau is reached, which does not lead to infinite telomeres (24-26). This feedback mechanism controls the number of telomere-binding protein, and leads to changes in telomere accessibility (17).

The growth behavior of the cells was observed. It was noted that there was only a slight reduction in cell growth after telomerase inhibition with plasmids 2, 6 and 7 .
However, telomerase inhibition had no significant effect on cell growth. These results differed from a study of mutants with hTER-transduced melanoma cells and bladder carcinoma cells. Telomerase inhibition led to a rapid growth arrest or even apoptosis (16). This may have been due to the lack of downregulation of telomerase in the telomeraseinhibitory plasmid-transduced cells. Another explaination for why the telomerase-inhibitory plasmids resulted in no significant inhibition of growth could be that the cells were able to quickly switch to an ALT mechanism. Compared with the controls, the cell morphology remained unchanged in Barrett's cells transduced with the telomerase inhibitors. The cells after transduction with the telomerase inhibitors were neither senescent nor apoptotic. In constrast, transduction with telomerase inhibitors in epithelial keratinocytes resulted in a strong inhibition of growth with several senescent and apoptotic-looking cells observed (22). This difference could arise from genetic alterations. In addition to exogenous overexpression of hTERT, there were no further genetic alterations noted in the esophageal keratinocytes. Inactivation of the tumor-suppressor p16 was noted in Barrett's cells, which is an important component for the activation of replicative senescence (27).

A conceptual model of recombination is that it occurs in ALT-positive cells by an exchange of telomeric sequences between sister chromatids or chromosomes, since it was observed that sister chromatid exchanges in ALT cells occur at a higher frequency when compared to telomerase-positive cells $(28,29)$.

Since $85 \%$ of immortalized and tumor cells are telomerase-positive, this is the most common telomere maintenance mechanism (4). This strong presence in human tumors makes this enzyme an attractive target for the development of new cancer therapeutics. Several telomerase inhibitors have been developed to eliminate telomerase in the hope of telomerase-positive tumor cells undergoing a shortening of telomeres, or the rupture of the T-loop structure to promote senescence or apoptosis. Telomerase induces a high proliferation rate not only in tumor cells, but also in 
normal tissues, such as hematopoietic stem and germ line cells. Thus, telomerase has the attractiveness of the target in question (30). Telomerase and ALT in the same cell can be active. ALT can be induced even after telomerase inhibition, reducing the attractiveness of telomerase as a therapeutic target. This was demonstrated in immortalized esophageal keratinocytes (31). A switch from telomerase to an ALT mechanism was also observed in esophageal keratinocytes after genetic telomerase inhibition (22).

In summary, our findings suggest that an alternative lengthening of telomere (ALT) mechanism was induced by telomerase inhibitors in Barrett's cells. In addition, the telomerase inhibitors may have high potency in the treatment of Barrett's esophagus. However, further studies and analyses are warranted.

\section{References}

1. Harley CB, Futcher AB and Greider CW: Telomeres shorten during ageing of human fibroblasts. Nature 345: 458-460, 1990.

2. Hahn WC and Meyerson M: Telomerase activation, cellular immortalization and cancer. Ann Med 33: 123-129, 2001.

3. Wright WE and Shay JW: Cellular senescence as a tumorprotection mechanism: the essential role of counting. Curr Opin Genet Dev 11: 98-103, 2001.

4. Kim NW, Piatyszek MA, Prowse KR, et al: Specific association of human telomerase activity with immortal cells and cancer. Science 266: 2011-2015, 1994.

5. Schaetzlein S, Lucas-Hahn A, Lemme E, et al: Telomere length is reset during early mammalian embryogenesis. Proc Nat Acad Sci USA 101: 8034-8038, 2004.

6. Dahse R, Fiedler W and Ernst G: Telomeres and telomerase: biological and clinical importance. Clin Chem 43: 708-714, 1997.

7. Allsopp RC, Cheshier S and Weissman IL: Telomere shortening accompanies increased cell cycle activity during serial transplantation of hematopoietic stem cells. J Exp Med 193: 917-924, 2001.

8. Son NH, Murray S, Yanovski J, et al: Lineage-specific telomere shortening and unaltered capacity for telomerase expression in human T and B lymphocytes with age. J Immunol 165: 1191-1196, 2000.

9. Masutomi K, Yu EY, Khurts S, et al: Telomerase maintains telomere structure in normal human cells. Cell 114: 241-253, 2003.

10. Hayflick L and Moorhead PS: The serial cultivation of human diploid cell strains. Exp Cell Res 25: 585-621, 1961.

11. Hahn WC and Weinberg RA: Rules for making human tumor cells. N Engl J Med 347: 1593-1603, 2002.

12. Opitz OG, Suliman Y, Hahn WC, et al: Cyclin D1 overexpression and p53 inactivation immortalize primary oral keratinocytes by a telomerase-independent mechanism. J Clin Invest 108: 725-732, 2001.

13. Goessel G, Quante M, Hahn WC, et al: Creating oral squamous cancer cells: a cellular model of oral-esophageal carcinogenesis. Proc Natl Acad Sci USA 102: 15599-15604, 2005.
14. Heeg S, Hirt N, Queisser A, et al: EGFR overexpression induces activation of telomerase via PI3K/AKT-mediated phosphorylation and transcriptional regulation through Hif1-alpha in a cellular model of oral-esophageal carcinogenesis. Cancer Sci 102: 351-360, 2011

15. Lansdorp PM, Verwoerd NP, van de Rijke, et al: Heterogeneity in telomere length of human chromosomes. Hum Mol Genet 5: $685-691,1996$.

16. Li S, Rosenberg JE, Donjacour, AA, et al: Rapid inhibition of cancer cell growth induced by lentiviral delivery and expression of mutant-template telomerase RNA and anti-telomerase shortinterfering RNA. Cancer Res 64: 4833-4840, 2004.

17. Pickett HA, Cesare AJ, Johnston RL, et al: Control of telomere length by a trimming mechanism that involves generation of t-circles. EMBO J 28: 799-809, 2009.

18. McEachern MJ and Blackburn EH: Runaway telomere elongation caused by telomerase RNA gene mutations. Nature 376: 403-409, 1995.

19. Singer MS and Gottschling DE: TLC1: template RNA component of Saccharomyces cerevisiae telomerase. Science 266: 404-409, 1994.

20. Yu GL, Bradley JD, Attardi LD, et al: In vivo alteration of telomere sequences and senescence caused by mutated Tetrahymena telomerase RNAs. Nature 344: 126-132, 1990.

21. Bryan TM, Englezou A, Gupta J, et al: Telomere elongation in immortal human cells without detectable telomerase activity. EMBO J 14: 4240-4248, 1995.

22. Döbele M, von Werder A, Fulda C, et al: Inhibition of telomerase by mutant template telomerase RNA and anti-telomerase short interfering RNA induces ALT in immortalized human epithelial cells. Z Gastroenterol 44: P281, 2006.

23. Cristofari $\mathrm{G}$ and Lingner J: Telomere length homeostasis requires that telomerase levels are limiting. EMBO J 25: 565-574, 2006.

24. Bianchi A and Shore D: How telomerase reaches its end: mechanism of telomerase regulation by the telomeric complex. Mol Cell 31: 153-165, 2008.

25. Smogorzewska A and de Lange T: Regulation of telomerase by telomeric proteins. Annu Rev Biochem 73: 177-208, 2004.

26. Smogorzewska A, van Steensel B, Bianchi A, et al: Control of human telomere length by TRF1 and TRF2. Mol Cell Biol 20: 1659-1668, 2000.

27. Collado M, Blasco MA and Serrano M: Cellular senescence in cancer and aging. Cell 130: 223-233, 2007.

28. Bechter OE, Zou Y, Walker W, et al: Telomeric recombination in mismatch repair deficient human colon cancer cells after telomerase inhibition. Cancer Res 64: 3444-3451, 2004.

29. Londono-Vallejo JA, Der-Sarkissian H, Cazes L, et al: Alternative lengthening of telomeres is characterized by high rates of telomeric exchange. Cancer Res 64: 2324-2327, 2004.

30. Broccoli D, Young JW and de Lange T: Telomerase activity in normal and malignant hematopoietic cells. Proc Natl Acad Sci USA 92: 9082-9086, 1995.

31. Von Werder A: Immortalized human esophageal squamous epithelial cells maintain their telomerases by either telomerase or ALT in a cell cycle-dependent fashion. http://www.biomedsearch. com/sci/immortalized-human-esophageal-squamous-epithelial/ 0040149415.html, 2007. 